Conclusions The extension of the follow-up confirms an increased risk for lymphatic and haemopoietic cancers, particularly leukemia, in the Seveso population.

\title{
Lymphatic neoplasms
}

109 LYMPHOHEMOPIETIC NEOPLASMS AND DIOXIN EXPOSURE IN THE SEVESO COHORT 30 YEARS AFTER THE ACCIDENT (1977-2006)

Angela Cecilia Pesatori, ${ }^{1}$ Dario Consonni, ${ }^{2}$ Raquel Carace, ${ }^{2}$ Raffaella Sindaco, ${ }^{2}$ Pier Alberto Bertazzi' ' 'Università di Milano, Milano, Italy; ${ }^{2}$ IRCCS Fondazione Ca' Granda, Milano, Italy

\subsection{6/oemed-2011-100382.109}

Objectives The Seveso accident caused the contamination of a large inhabited area by 2,3,7,8-tetrachlorodibenzodioxin. Three zones with decreasing soil TCDD levels were delimited: A, B and R. Persons ever living in the contaminated zones and in a surrounding non-contaminated area were followed-up to evaluate long-term effects. The main finding of the mortality (1976-2001) and cancer incidence studies (1977-1991) was an increase in lymphohemopoietic cancers in zones $\mathrm{A}$ and $\mathrm{B}$. We report preliminary results of the extension of the follow-up to 30 years after the accident for lymphoemopietic cancers.

Methods Incident cases were ascertained through the hospital discharge registration system of the Lombardy region and medical records were reviewed. RR and 95\% CI were estimated with Poisson regression techniques controlling for age, gender and calendar period using the non-contaminated area as reference.

Results The incidence of all lymphohemopietic cancers was increased in zone A ( 6 cases, RR $=1.2 ; 95 \%$ CI 0.5 to 2.7 ) and B (47 cases; $R R=1.5 ; 95 \%$ CI 1.1 to 2.0 ). Non-Hodgkin's lymphomas were modestly increased in zone $B$, whereas the RR for all leukaemias was 2.0 (95\% CI 1.2 to 3.4). 5 cases were lymphatic leukaemia ( $R R=1.695 \%$ CI 0.6 to 4.1 ); 10 myeloid leukaemias showed a RR of 2.1 (95\% CI 1.04 to 4.15). All lymphatic leukaemias occurred after 20 years since the accident $(R R=4.0 ; 95 \%$ CI 1.4 to 11.6). Myeloid leukaemia did not show a consistent pattern across different categories of time since the accident. 